\title{
Development of an Unstructured Navier-Stokes Solver For Hypersonic Nonequilibrium Aerothermodynamics
}

\author{
Leonardo C. Scalabrin* and Iain D. Boyd ${ }^{\dagger}$ \\ Department of Aerospace Engineering, University of Michigan, Ann Arbor, MI, 48109, USA
}

\begin{abstract}
Numerical simulations of a spacecraft at reentry conditions are presented. The fluid at such conditions is modeled as a reacting gas in thermal and chemical nonequilibrium. The reacting gas is modeled using a standard finite rate chemistry model for air. A twotemperature model is used to account for the thermal nonequilibrium effects. The finitevolume method is used to solve the set of differential equations on unstructured meshes. Numerical fluxes between the cells are discretized using a modified Steger-Warming Flux Vector Splitting (FVS) which has low dissipation and is appropriate to calculate boundary layers. The scheme switches back to the original Steger-Warming FVS near shock waves. A point implicit method is used to perform the time march. The numerical results for heat transfer are compared to available experimental data. The influence of the method used to switch between schemes on the results is assessed.
\end{abstract}

\section{Introduction}

$\mathrm{T}$ HERE is a renewed interest in atmospheric reentry of spacecraft in order to achieve goals of returning to the Moon and going to Mars. Accurate prediction of aerodynamic coefficients and of heat transfer along the reentry trajectory are some of the main goals for aerothermodynamic research on reentry problems. Due to extreme variations in the environment and velocity, a reentry trajectory covers a broad range of flow conditions. The flow can vary from hypersonic to subsonic flow. In addition, the flow can also vary from a non-continuum to a continuum flow. More importantly, at a single point in the trajectory, the flow can be of mixed types around the spacecraft. Therefore, there is a need for codes that can handle simultaneously different types of flow. While there is a myriad of codes that can simulate flows in which there are supersonic and subsonic regions, that is not the case when the flow contains continuum and non-continuum regions.

The simulation of flows with mixed continuum and non-continuum regions is a current area of research. ${ }^{1-3}$ The use of Information Preserving methods is one methodology on such a complex problem. ${ }^{1}$ Currently, a different approach allowing for communication between a standard Navier-Stokes (NS) code and a Direct Simulation Monte-Carlo (DSMC) code is being developed. ${ }^{4}$ It is clear that the quality of any code designed to simulate a flow of mixed type will depend very strongly on how well the code can simulate flows of a single type.

Most NS codes can not accurately simulate flows in thermal and chemical nonequilibrium, a condition commonly seen in reentry problems. This fact prompted the implementation of a state of the art NS code capable of simulating flow in those demanding conditions. The code models the fluid as a reacting gas in thermal and chemical nonequilibrium. A two-temperature model is used to account for the nonequilibrium between the vibrational and the translational-rotational modes. ${ }^{5-7}$ The set of equations is solved using a finite-volume method on unstructured meshes. ${ }^{8}$ One main reason for using unstructured meshes is the desire

${ }^{*}$ Graduate Studante, Student Member AIAA.

${ }^{\dagger}$ Professor, Associate Fellow AIAA 
to develop a general CFD module to be used in a hybrid continuum/non-continuum code. The code is able to handle meshes made of triangles, quadrilaterals, tetrahedra, hexahedra, pyramids and prisms. The inviscid fluxes between the mesh volumes are discretized using a modified form of the Steger-Warming Flux Vector Splitting (FVS) scheme ${ }^{9,10}$ which is less dissipative and adequate to calculate boundary layers. The scheme switches back to the original form of Steger-Warming near shock waves. The viscous terms are calculated using the values of properties at the cell centers and at the nodes. ${ }^{11}$ The time integration is performed using a point-implicit method. ${ }^{12-14}$

The paper focuses only on axisymmetric results. The mathematical formulation of the physical model used as well as the numerical formulation is presented. Assessment is made of the switching between the original and modified Steger-Warming FVS schemes and the impact in the amount of dissipation added to stabilize the method. It is found that axisymmetric flow simulations using unstructured meshes can cause unexpected oscillations close to the symmetry line. Finally, numerical results of axisymmetric flow over a laboratory model of a Mars entry vehicle at hypersonic conditions are presented and compared to available experimental results.

\section{Mathematical Formulation}

The flow is modeled assuming that the continuum approximation is valid and that the fluid is non-ionized. Furthermore, it is assumed that the rotational and translational energy modes of all species can be described by a single temperature $T$ and that the vibrational energy mode of all species can be described by a single temperature $T_{v}$. With those approximations, the conservation equations for the two-dimensional system can be written $\operatorname{as}^{5-7,15}$

$$
\frac{\partial Q}{\partial t}+\frac{\partial\left(E-E_{v}\right)}{\partial x}+\frac{\partial\left(F-F_{v}\right)}{\partial y}=S_{c v}
$$

where

$$
Q=\left\{\begin{array}{c}
\rho_{1} \\
\vdots \\
\rho_{n s} \\
\rho u \\
\rho v \\
E \\
E_{v}
\end{array}\right\} \quad \text { and } \quad S_{c v}=\left\{\begin{array}{c}
\dot{w}_{1} \\
\vdots \\
\dot{w}_{n s} \\
0 \\
0 \\
0 \\
\dot{w}_{v}
\end{array}\right\}
$$

are the vector of conserved variables and the vector of source terms, respectively. In these expressions, $\rho_{1} \cdots \rho_{n s}$ are the species densities, $u$ and $v$ are the bulk velocity components, $E$ and $E_{v}$ are the total and the vibrational energy per unit volume of mixture, respectively.

The inviscid and diffusive flux vector components in the $x$ direction are given by

$$
E=\left\{\begin{array}{c}
\rho_{1} u \\
\vdots \\
\rho_{n s} u \\
\rho u^{2}+p \\
\rho u v \\
(E+p) u \\
E_{v} u
\end{array}\right\} \quad \text { and } \quad E_{v}=\left\{\begin{array}{c}
-\rho_{1} u_{d 1} \\
\vdots \\
-\rho_{n s} u_{d n s} \\
\tau_{x x} \\
\tau_{x y} \\
\tau_{x x} u+\tau_{x y} v-\left(q_{t x}+q_{r x}+q_{v x}\right)-\sum\left(\rho_{s} h_{s} u_{d s}\right) \\
-q_{v x}-\sum\left(\rho_{s} e_{v s} u_{d s}\right)
\end{array}\right\}
$$

where $p$ is the pressure, $\tau_{i j}$ are the viscous stress components, $q_{t i}, q_{r i}$ and $q_{v i}$ are the translational, rotational and vibrational heat fluxes in the $i$-th direction. Moreover, $h_{s}$ is the species enthalpy, $u_{d s}$ is the species

$$
2 \text { of } 18
$$


diffusion velocity in the $x$-direction and $e_{v s}$ is the species vibrational energy per unit mass. The flux vectors in the other directions are similar. The following subsections provide more details on the modeling of the terms.

\section{A. Transport Models}

The viscous stresses are modeled assuming a Newtonian fluid for which, using Stokes' hypothesis,

$$
\tau_{i j}=\mu\left(\frac{\partial u_{j}}{\partial x_{i}}+\frac{\partial u_{i}}{\partial x_{j}}\right)+\lambda \nabla \vec{u} \delta_{i j} \quad, \quad \lambda=-\frac{2}{3} \mu \quad,
$$

where $\mu$ is the mixture coefficient of viscosity.

The heat fluxes are modeled according to Fourier's law as

$$
\vec{q}_{t, r, v}=-\kappa_{t, r, v} \nabla T_{\mathrm{t}, \mathrm{r}, \mathrm{v}}
$$

where $\kappa_{t, r, v}$ are the mixture thermal conductivity for each energy mode. The species mass diffusion fluxes are modeled using Fick's law as

$$
\rho_{s} \vec{u}_{d s}=-\rho D_{s} \nabla\left(\frac{\rho_{s}}{\rho}\right)
$$

where $D_{s}$ is the species diffusion coefficients.

\section{B. Thermodynamic Properties}

The mixture pressure $p$ is obtained using Dalton's law of partial pressures and the perfect gas law for each species. Thus

$$
p=\sum_{s} p_{s}=\sum_{s} \rho_{s} \frac{R}{M_{s}} T=\rho \bar{R} T
$$

where $\bar{R}=\sum_{s} \frac{\rho_{s}}{\rho} \frac{R}{M_{s}}, R$ is the universal gas constant and $M_{s}$ is the species molecular weight. One should observe that $T$ is the translational-rotational temperature. The total energy per unit volume of mixture is given by

$$
E=\sum_{s} \rho_{s} C_{V s} T+\frac{1}{2} \rho|\vec{u}|^{2}+\sum_{s} \rho_{s} e_{v s}+\sum_{s} \rho_{s} h_{s}^{o}
$$

where $h_{s}^{0}$ is the species enthalpy of formation and $C_{V s}$ is the translational-rotational specific heat at constant volume. It is given by

$$
C_{V s}=C_{V s}^{(t r)}+C_{V s}^{(r o t)}
$$

This work assumes constant values for $C_{V s}^{(t r)}$ and $C_{V s}^{(r o t)}$ as

$$
C_{V s}^{(t r)}=\frac{3}{2} \frac{R}{M_{s}} \quad \text { and } \quad C_{V s}^{(r o t)}=\frac{R}{M_{s}} \quad .
$$

The species vibrational energy per unit mass is modeled using a harmonic oscillator as

$$
e_{v s}=\frac{R}{M_{s}} \frac{\theta_{v s}}{\exp \left(\theta_{v s} / T_{v}\right)-1}
$$

and the vibrational specific heat at constant volume is calculated by

$$
C_{V s}^{(v i b)}=\frac{\partial e_{v s}}{\partial T_{v}}
$$

Finally, the mixture vibrational energy per unit volume is given by

$$
E_{v}=\sum_{s} \rho_{s} e_{v s}
$$




\section{Transport Properties}

The mixture transport properties are modeled using Wilke's semi-empirical mixing rule, ${ }^{16}$ for which

$$
\mu=\sum_{s} \frac{X_{s} \mu_{s}}{\phi_{s}} \quad \text { and } \quad \kappa=\sum_{s} \frac{X_{s} \kappa_{s}}{\phi_{s}}
$$

where $X_{s}$ is the species molar fraction, $\mu_{s}$ is the species coefficient of viscosity and $\kappa_{s}$ is the species thermal conductivity. It should be noted that there is a $\kappa$ for each energy mode: translational, rotational and vibrational. The term $\phi_{s}$ is given by

$$
\phi_{s}=\sum_{r} X_{r}\left[1+\sqrt{\frac{\mu_{s}}{\mu_{r}}}\left(\frac{M_{r}}{M_{s}}\right)^{1 / 4}\right]^{2}\left[\sqrt{8\left(1+\frac{M_{s}}{M_{r}}\right)}\right]^{-1} .
$$

The species viscosities are calculated using Blottner's model ${ }^{17}$ as

$$
\mu_{s}=0.1 \exp \left[\left(A_{s} \ln T+B_{s}\right) \ln T+C_{s}\right],
$$

where $A_{s}, B_{s}$ and $C_{s}$ are constants determined for each species. The species thermal conductivities are determined using Eucken's relation ${ }^{18}$ as

$$
\kappa_{t s}=\frac{5}{2} \mu_{s} C_{V s}^{(t r)} \quad, \quad \kappa_{r s}=\mu_{s} C_{V s}^{(r o t)} \quad \text { and } \quad \kappa_{v s}=\mu_{s} C_{V s}^{(v i b)} \quad .
$$

The mass diffusion coefficient for each species, $D_{s}$, is replaced by a single binary coefficient $D$. It can be obtained assuming a constant Lewis number, Le, by

$$
D=\frac{L e \kappa}{\rho C_{p}}
$$

where $\kappa$ is the mixture rotational-translational thermal conductivity and $C_{p}$ is the mixture translationalrotational specific heat at constant pressure.

\section{Chemical Model}

This work assumes a standard finite-rate chemistry model for reacting air. The reactions are

$$
\begin{gathered}
N_{2}+M \rightleftharpoons 2 N+M \\
O_{2}+M \rightleftharpoons 2 O+M \\
N O+M \rightleftharpoons N+O+M \\
N_{2}+O \rightleftharpoons N O+N \\
N O+O \rightleftharpoons O_{2}+N
\end{gathered}
$$

and they can be represented generically as

$$
\sum \nu_{s}^{\prime}[S] \rightleftharpoons \sum \nu_{s}^{\prime \prime}[S]
$$

One should observe that $M$ in these reactions stands for any collision partner. Hence, the model has 17 reactions. The chemical production rate of species $s$ in reaction $\mathrm{k}$ is given by

$$
\begin{gathered}
\dot{w}_{s k}=\left(\nu_{s k}^{\prime \prime}-\nu_{s k}^{\prime}\right)\left[k_{f k} \prod_{j} X_{j}^{\nu_{j k}^{\prime}}-k_{b k} \prod_{j} X_{j}^{\nu_{j k}^{\prime \prime}}\right] \\
4 \text { of } 18
\end{gathered}
$$


and the source terms in the species conservation equations are given by

$$
\dot{w}_{s}=M_{s} \sum_{k} \dot{w}_{s k}
$$

The forward and backward reaction rates are affected by the level of nonequilibrium in the flow. To account for that effect, Park's two-temperature model ${ }^{7}$ is used. In that model,

$$
k_{f k}=C_{f k} T_{P}^{\eta_{k}} \exp \left(-\theta_{k} / T_{P}\right),
$$

where

$$
T_{P}=\sqrt{T T_{v}}
$$

and $C_{f} k, \eta_{k}$ and $\theta_{k}$ are constants. ${ }^{7}$ The backward reaction rate is obtained from

$$
k_{b k}\left(T_{P}\right)=\frac{k_{f k}\left(T_{P}\right)}{K_{e q}(T)},
$$

with the equilibrium constant given by curve fits ${ }^{7}$ of the form

$$
K_{e}(T)=\exp \left[A_{1}\left(\frac{T}{10000}\right)+A_{2}+A_{3} \ln \left(\frac{10000}{T}\right)+A_{4}\left(\frac{10000}{T}\right)+A_{5}\left(\frac{10000}{T}\right)^{2}\right],
$$

where the coefficients $A_{i}$ are a function of the local number density. The values for $A_{i}$ are interpolated from the values given in Ref. 7 for number densities within the range of data. For number densities outside the range given in that reference, the tabulated values for the maximum and minimum number densities are used accordingly.

\section{E. Relaxation Model}

The source term in the vibrational energy equation is modeled as

$$
\dot{w}_{v}=\sum_{s} Q_{s}^{v}+\sum_{s} Q_{s}^{t-v}
$$

where $Q_{s}^{v}$ is the rate of production of vibrational energy due to reactions, which can be written as

$$
Q_{s}^{v}=\dot{w}_{s} e_{v s}
$$

The term $Q_{s}^{t-v}$ is the energy exchange rate between the vibrational and the translational-rotational energy modes. It is assumed that the vibrational-translational and the vibrational-rotational energy exchange rates can be modeled by a single vibrational-translational/rotational energy exchange rate. The energy exchange rate is modeled using the Landau-Teller model ${ }^{18}$ as

$$
Q_{s}^{t-v}=\rho_{s} \frac{e_{v s}^{*}-e_{v s}}{<\tau_{s}>},
$$

where the molar averaged Landau-Teller relaxation time ${ }^{5}$ is written as

$$
<\tau_{s}>=\frac{\sum_{r} X_{r}}{\sum_{r} X_{r} / \tau_{s r}}
$$

where $X_{r}$ is the molar fraction of species $r$. The Landau-Teller inter-species relaxation time, $\tau_{s r}$, is modeled by $^{19}$

$$
\begin{gathered}
\tau_{s r}=\frac{101325}{p} \exp \left[A_{s r}\left(T^{-1 / 3}-0.015 \mu_{s r}^{1 / 4}\right)-18.42\right] \\
A_{s r}=1.16 \cdot 10^{-3} \mu_{s r}^{1 / 2} \theta_{v s}^{4 / 3} \\
\mu_{s r}=\frac{M_{s} M_{r}}{M_{s}+M_{r}}
\end{gathered}
$$




\section{Numerical Formulation}

The finite-volume method ${ }^{8}$ is obtained by integrating the set of equations in a generic mesh cell. Assuming axisymmetry, it follows that ${ }^{15}$

$$
r_{i} A_{i} \frac{\partial Q_{i}}{\partial t}=-\sum_{j}\left(\vec{F}_{j}-\vec{F}_{d j}\right) \cdot \vec{n}_{j} r_{j} s_{j}+r_{i} A_{i} S_{c v i}+A_{i} S_{a i}=R_{i}
$$

where $\vec{F}=E \vec{i}+F \vec{j}+G \vec{k}$ is the inviscid flux at the face, $\vec{F}_{d}=E_{d} \vec{i}+F_{d} \vec{j}+G_{d} \vec{k}$ is the diffusive flux at the face, $S_{c v i}$ is the chemistry and non-equilibrium source term, $S_{a i}$ is the source term that arises from the integration being performed assuming axisymmetry, $A_{i}$ is the area of the i-th cell, $\vec{n}$ is the normal vector to the $\mathrm{j}$-th face and $s_{j}$ is the length of the $\mathrm{j}$-th face. The radial coordinate of the $\mathrm{i}$-th cell, $r_{i}$, is measured from its centroid. The radial coordinate of the j-th face, $r_{j}$, is measured from its mid-point. The term $S_{a i}$ can be written as

$$
S_{a i}=\left\{\begin{array}{c}
0 \\
\vdots \\
0 \\
0 \\
p-2 \mu \frac{v}{r_{i}}-\lambda \vec{\nabla} \cdot \vec{u} \\
0 \\
0
\end{array}\right\}
$$

In the axisymmetric formulation, $\vec{\nabla} \cdot \vec{u}$ in the previous expression and in the stress tensor is given by

$$
\vec{\nabla} \cdot \vec{u}=\frac{\partial u}{\partial x}+\frac{\partial v}{\partial y}+\frac{v}{r}
$$

The implementation of axisymmetric terms on codes using unstructured meshes presents some additional complications. The presence of the source terms can make the code unable to preserve a simple freestream flow. This is due to the non-cancellation of pressure forces acting on the cell. This problem can be easily solved by substituting the cell area multiplying the axisymmetric source term $\left(A_{i}\right)$ by a modified area $\left(A_{a i}\right)$ given by

$$
A_{a i}=\sum_{j} n_{j y} s_{j} r_{j}
$$

which still is an approximation to the area of the cell.

\section{A. Spatial Discretization}

The inviscid flux vector at the $j$-th face is discretized using the Steger-Warming FVS scheme. ${ }^{10}$ It uses the homogeneous property of the inviscid flux vector,

$$
\vec{F} \cdot \vec{n}=F_{n}=\frac{d F_{n}}{d Q} Q=A Q
$$

where $F_{n}$ is the normal flux at the $\mathrm{j}$-th face. The matrix $A$ is called the Jacobian of the inviscid flux. It can be diagonalized by the matrices of its eigenvectors $S$ and $S^{-1}$ as

$$
A=S^{-1} \Lambda S
$$

and then separated into positive and negative parts

$$
\begin{aligned}
& A^{+}=S^{-1} \Lambda^{+} S \text { and } A^{-}=S^{-1} \Lambda^{-} S, \\
& \text { American Institute of Aeronautics and Astronautics }
\end{aligned}
$$


where the matrices $\Lambda$ are the diagonal matrices of the eigenvalues of the Jacobian. The Steger-Warming scheme uses this split of the Jacobian to separate the flux on a downstream and on an upstream flux in relation to the face orientation as

$$
\vec{F}_{j} \cdot \vec{n}_{j}=A_{j} Q_{j}=\left(A_{i}^{+} Q_{i}+A_{n b}^{-} Q_{n b}\right) \quad,
$$

where $i$ and $n b$ indicate the cells on the left and right side of the face, respectively. The expressions for the matrices of the particular set of equations solved in this work can be found in Ref. 15. The eigenvalues of the Jacobian are calculated by ${ }^{20}$

$$
\lambda^{ \pm}=\frac{1}{2}\left(\lambda \pm \sqrt{\lambda^{2}+\epsilon^{2}}\right)
$$

where $\epsilon$ is supposed to be a small number used only to correct the sonic glitch problem. ${ }^{20}$ However, it is very common practice to use it to add numerical dissipation to the scheme.

The Steger-Warming FVS is too dissipative and it cannot calculate boundary layer profiles accurately. ${ }^{9}$ Therefore, a modified Steger-Warming FVS scheme ${ }^{9}$ is used at boundary layers. The modification simply involves calculating the matrices $A^{+}$and $A^{-}$using an average of the states on the left and right sides of the face,

$$
\vec{F}_{j} \cdot \vec{n}_{j}=A_{j} Q_{j}=\left(A_{j+}^{+} Q_{i}+A_{j-}^{-} Q_{n b}\right)
$$

where the subscripts $j+$ and $j$ - indicate that the Jacobians are no longer evaluated at $i$ or $n b$, but at average states

$$
Q_{j+}=\frac{Q_{i}+Q_{n b}}{2} \quad \text { and } \quad Q_{j-}=\frac{Q_{i}+Q_{n b}}{2} .
$$

This approach is very efficient and gives good results for boundary layers. However, it is not suitable in the vicinity of strong shock waves. Close to shock waves, the method needs to be switched back to the original Steger-Warming scheme which is more dissipative. A popular approach is to use a pressure switch of the form $^{21}$

$$
Q_{j+}=(1-w) Q_{i}+w Q_{n b} \quad \text { and } \quad Q_{j-}=(1-w) Q_{n b}+w Q_{i}
$$

where

$$
w=\frac{1}{2} \frac{1}{(0.5 \nabla p)^{2}+1} \quad \text { and } \quad \nabla p=\frac{\left|p_{i}-p_{n b}\right|}{\min \left(p_{i}, p_{n b}\right)}
$$

The modified Steger-Warming FVS scheme corresponds to $w=(1-w)=0.5$, which happens for small values of $\nabla p$. The original Steger-Warming FVS scheme is recovered for $w=0$ and $(1-w)=1$ which happens for large values of $\nabla p$.

It will be shown in the results section that this switch in fact does not switch back to the original StegerWarming scheme even for very strong shock waves. This causes oscillations in the results and it demands additional dissipation which is provided by unphysically increasing the values of $\epsilon$ in the eigenvalues of the Jacobian. In Ref. $21, \epsilon=0.3 a$ is used where $a$ is the speed of sound, a very large number in stagnation regions. The unphysical values of $\epsilon$ need to be switched back to zero close to the body. ${ }^{21}$ Even though that can be easily done in a structured mesh, this is rather cumbersome in an unstructured mesh. As the problem starts from the switching between methods, a new switch of the form

$$
w=0.25-0.25 \operatorname{sign}\left(\nabla^{* *} p-0.3\right)\left(1-\exp \left(-100\left|\nabla^{* *} p-0.3\right|\right)\right)
$$

where

$$
\nabla^{* *} p=\max \left(\nabla^{*} p_{i}, \nabla^{*} p_{n b}\right) \quad \text { and } \quad \nabla^{*} p_{i}=\max \left(\nabla p_{1}, \ldots, \nabla p_{n f}\right)
$$

is used in this work. In the previous relation, $n f$ is the number of faces of the i-th cell. This switch does switch to the original Steger-Warming FVS at shock waves and, therefore, allows the use of a constant, small value for $\epsilon$. The comparison between methods is presented in the results section. 
The viscous terms are calculated using the values of properties at the cell centers and at the nodes. ${ }^{11}$ The property values at the nodes are calculated using a simple average of the values of the cells that share that node. Use of this method increases the stencil used in the derivative calculations which avoids loss of accuracy when using unstructured meshes. ${ }^{11}$

The source terms are evaluated as

$$
S_{c v i}=S_{c v}\left(Q_{i}\right) \quad \text { and } \quad S_{a i}=S_{a}\left(Q_{i}\right) .
$$

Caution must be used when including chemistry in a CFD code. The forward and backward rate of reactions can be very large numbers depending on the temperature in the cell. In this work, for the conditions simulated, the backward rate of reaction for small temperatures sometimes became too large due to very small values for the equilibrium constant $K c$. The equilibrium constant is therefore modified to

$$
K c^{\prime}=\max \left(10^{-25}, K c\right) \text {. }
$$

Another important modification that needs to be addressed in a CFD code is that species densities need to be positive at all times, even for very small numbers. A negative value for species density, even a very small negative number, changes the sign of the chemical source terms causing the code to diverge.

\section{B. Time March}

This work uses a point implicit method ${ }^{12-14}$ to perform the time march. The method used in this work is an extension of the DP-LUR method ${ }^{12}$ for unstructured grids. Starting from the backward Euler method one obtains

$$
\frac{A_{i} r_{i}}{\Delta t} \Delta Q_{i}^{n}=\left[-\sum_{j}\left(F_{n j}-F_{d n j}\right) s_{j} r_{j}+A_{i} r_{i} S_{c v i}+A_{a i} S_{a i}\right]^{n+1}=R_{i}^{n+1},
$$

where $R_{i}^{n+1}$ is called the residue at the i-th cell. Expanding the residue at time $n+1$ in terms of the residue at time $n$ it follows that

$$
\begin{aligned}
\frac{A_{i} r_{i}}{\Delta t} \Delta Q_{i}^{n}=R_{i}^{n}+ & {\left[-\sum_{j}\left(\left(\frac{\partial F_{n}}{\partial Q}\right)_{j} \Delta Q-\left(\frac{\partial F_{d n}}{\partial Q}\right)_{j} \Delta Q\right) s_{j} r_{j}+\right.} \\
& \left.A_{i} r_{i}\left(\frac{\partial S_{c v}}{\partial Q}\right)_{i} \Delta Q_{i}+A_{a i}\left(\frac{\partial S_{a}}{\partial Q}\right)_{i} \Delta Q_{i}\right]^{n} .
\end{aligned}
$$

Now, from the spatial discretization of the inviscid terms,

$$
\left(\frac{\partial F_{n}}{\partial Q}\right)_{j} \Delta Q=A_{j+}^{+} \Delta Q_{i}+A_{j-}^{-} \Delta Q_{n b}
$$

and one can similarly approximate the diffusive terms by ${ }^{22}$

$$
\left(\frac{\partial F_{d n}}{\partial Q}\right)_{j} \Delta Q=B_{j-}^{-} \Delta Q_{n b}-B_{j+}^{+} \Delta Q_{i}
$$

Thus, a line in the system of equations to be solved can be written as

$$
\begin{array}{r}
{\left[\frac{A_{i} r_{i}}{\Delta t}+\sum_{j}\left(A_{j+}^{+}+B_{j+}^{+}\right) s_{j} r_{j}-A_{i} r_{i}\left(\frac{\partial S_{c v}}{\partial Q}\right)_{i}-A_{a i}\left(\frac{\partial S_{a}}{\partial Q}\right)_{i}\right] \Delta Q_{i}+} \\
\sum_{j}\left(A_{j-}^{-}-B_{j-}^{-}\right) s_{j} r_{j} \Delta Q_{n b}=R_{i}^{n}
\end{array}
$$

8 of 18 
or, in a simpler form, as

$$
M \Delta Q_{i}+\sum_{j} N_{j} \Delta Q_{n b}=R_{i}
$$

The system of equations is solved iteratively using

$$
M \Delta Q_{i}^{k+1}=-\sum_{j} N_{j} \Delta Q_{n b}^{k}+R_{i} .
$$

Following Ref. 12, four iterations are taken in the iterative process. The code starts running with a very small $C F L$ number which is increased as the $L_{2}$ norm decreases. ${ }^{23}$ The maximum $C F L$ number of this method varies according to the freestream conditions ${ }^{12}$ and in fact, a good rule is to limit the maximum time step rather than the $C F L$ number. In this work, the maximum time step is limited to values ranging from $10^{-7} s$ to $10^{-6} s$.

\section{Results}

\section{A. Inviscid Axisymmetric Freestream Flow}

Due to the presence of strong source terms close to the symmetry axis, axisymmetric flow simulations can produce unphysical solutions if the source terms are not treated correctly. In order to assess the code behavior, a freestream flow in an axisymmetric domain is simulated. The flow properties are $M=7.9$, $p=1384 \mathrm{~Pa}, \mathrm{~T}=1130 \mathrm{~K}$ and the fluid is air. Figure 1 presents the mesh used in the test. The left edge is a freestream boundary, the top and right edges are outlet boundaries and the bottom edge is a symmetry boundary. The mesh does not use significant stretching in any direction and it is very smooth. However,

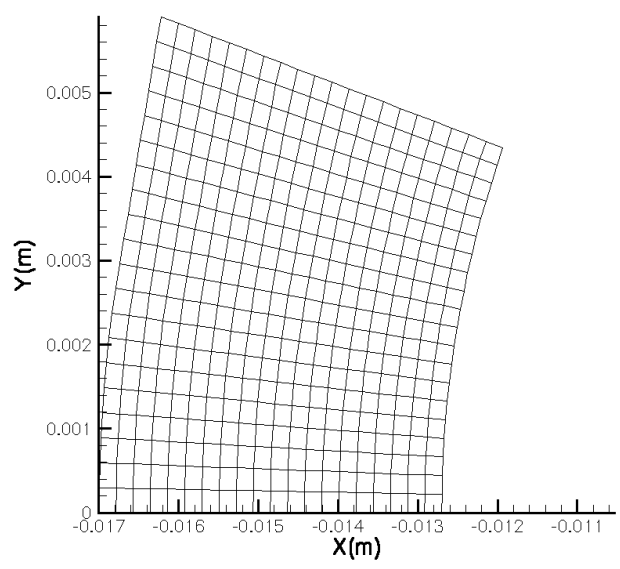

Figure 1. Mesh used to test the axisymmetric source terms.

even when using such a well-behaved mesh the axisymmetric source terms can lead to unphysical results. Figure 2(a) presents the contours of vertical velocity in the small domain simulated. Unphysical values for the vertical velocities are clearly displayed. The streamlines are straight lines due to the very high magnitude of the axial velocity. Fortunately, the problem is very easy to fix. Using another approximation for the cell area as given in Eq. 35 eliminates the problem, as can be observed in Fig. 2(b). 


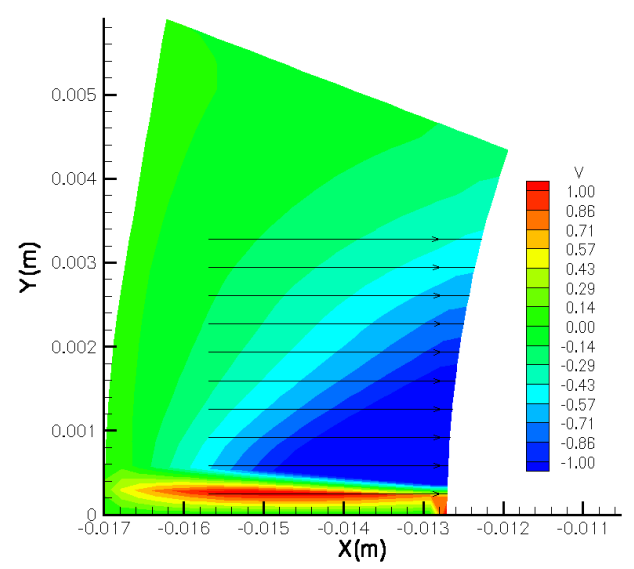

(a) Cell area.

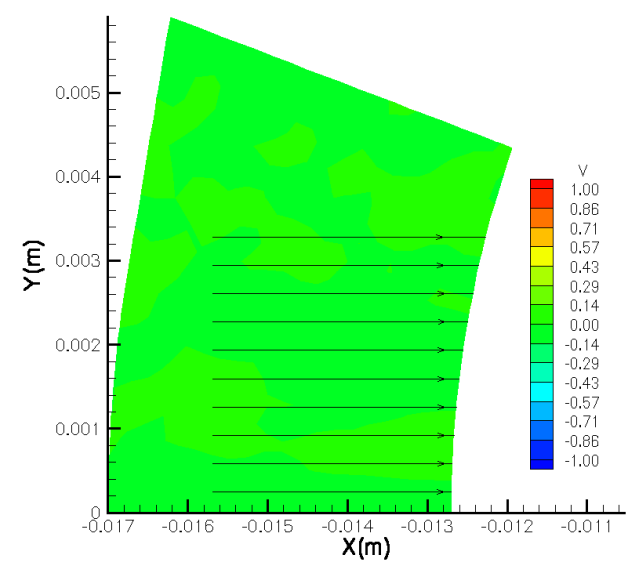

(b) Modified cell area.

Figure 2. Contour of vertical velocities $(\mathrm{m} / \mathrm{s})$.

\section{B. Assessment of Switching between Original and Modified Steger-Warming FVS}

In this work, two pressure switches are used to switch between the original and the modified Steger-Warming FVS schemes near shock waves. The motivation to develop a new pressure switch comes from difficulties encountered when trying to extend existing methodologies used in structured meshes to unstructured meshes. In particular, it was attempted to turn the eigenvalue limiter $\epsilon$ to zero following the recipes detailed in Ref. 21. The difficulties faced prompted the authors to question the need for a large value for $\epsilon$ in the first place. According to the original papers ${ }^{10,20} \epsilon$ should be a very small number, $\epsilon<<1$. This fact hinted that extra dissipation in the form of unphysical values for $\epsilon$ was being needed to keep the method stable. This could only happen if the modified Steger-Warming FVS was being used in the shock wave, because the original Steger-Warming FVS does not need any additional dissipation.

Figure 3(a) presents the calculated values of the pressure switch proposed in Ref. 21 as a function of the pressure jump across different strength normal shock waves. It is clear from this figure that the method would completely switch back to the original Steger-Warming FVS, which happens for $(1-w)=1$, only for a Mach number upstream of the shock larger than 4 . The proposed switch does a better job and the method is already the original Steger-Warming FVS around $M=1.1$. The situation in a real CFD computation is even worse, because a shock wave is not captured in just one cell. This smears out the pressure jump across many cells and it leads to smaller pressures jumps at each interface. The smaller pressure jumps may not be enough to make the pressure switch totally recover the original Steger-Warming scheme. This is clearly observed in Fig. 3(b), which shows the pressure jump across the cells on the stagnation line in a hypersonic flow simulation over a reentry vehicle at $M=7.9$. One can observe that the switch from Ref. 21 never switches back to the original Steger-Warming method because $(1-w)$ is always less than 1 . The switch proposed in this work reaches the value 1 as soon as the shock starts guaranteeing the use of the original Steger-Warming FVS in the shock region. It also drops to 0.5 immediately after the end of the shock enabling the use of the modified Steger-Warming FVS in the boundary layer. The use of the proposed pressure switch eliminates the need to change the value of $\epsilon$ according to the region or face orientation. A constant small value around 0.1 or smaller is now used. 


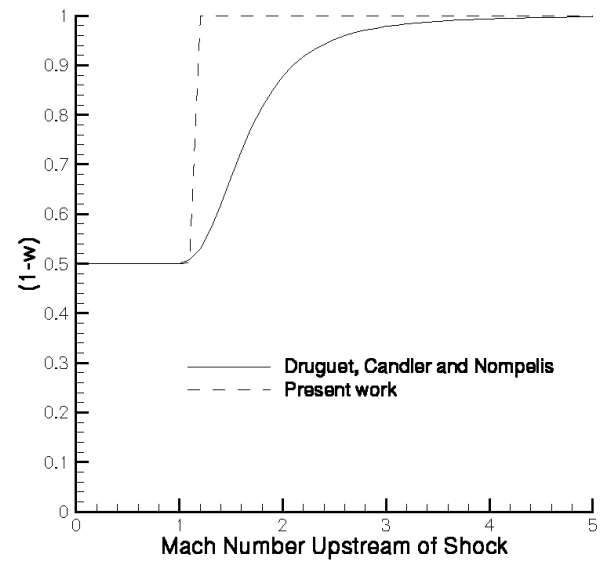

(a) Normal shock wave pressure jumps.

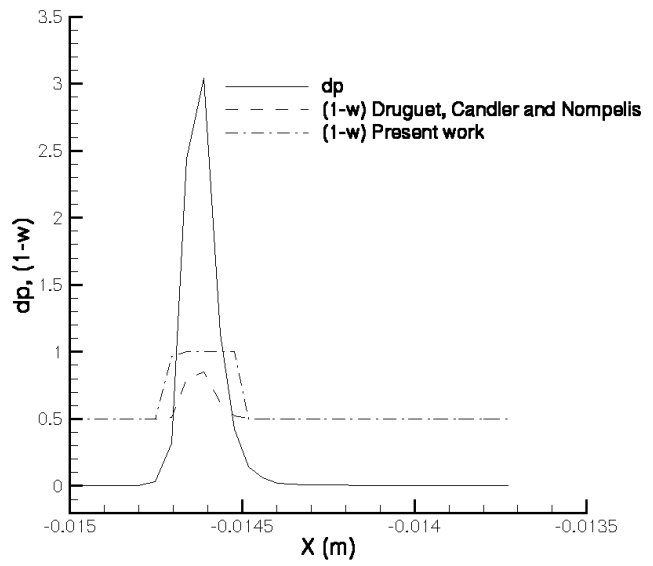

(b) Grid captured normal shock wave, $M_{\infty}=7.9$.

Figure 3. Pressure switch comparisons.

The impact of the switches in the amount of dissipation added to the method in the form of large values of $\epsilon$ is assessed by simulating the forebody of an experimental model of a Mars entry vehicle at thermochemical nonequilibrium. Detailed geometry information can be obtained in Refs. 24-26. The generated mesh has 26000 cells, 200 rows of cells in the direction normal to the body and 130 rows along the body. It can be observed in Fig. 4. The mesh is generated using the commercial software GAMBIT. Even though the mesh looks structured, the code treats it as an unstructured mesh. The freestream conditions for the flow are $u_{\infty}=5162 \mathrm{~m} / \mathrm{s}, T_{t \infty}=1113 \mathrm{~K}, \rho_{\infty}=5.7110^{-3} \mathrm{~kg} / \mathrm{m}^{3}, T_{t \infty}=T_{v \infty}, Y_{N_{2}}=0.7653$ and $Y_{O_{2}}=0.2347$. The wall temperature is assumed constant and equal to $T_{w}=300 \mathrm{~K}$. It is also assumed that $T_{t w}=T_{v w}$.

The heat transfer calculated using both switches is presented in Fig. 5. The smaller value of $\epsilon$, allowed by the modified pressure switch, gives numerical results in good agreement to the experimental results except for the stagnation point region. The pressure switch of Ref. 21, which demands additional dissipation in the form of a large value for $\epsilon$ overpredicts the heat transfer. That is due to the extra dissipation which causes the temperature close to the wall to be higher. This then increases the heat transfer to the wall because the wall temperature is fixed. It is not clear at this moment why the code is overpredicting the heat transfer at the stagnation point. It is expected that the heat transfer for a blunt body would be flat near to the stagnation point instead of the sudden rise seen in Fig. 5. Further numerical studies are needed to address this behavior. The use of a large value for $\epsilon$ also causes unphysical velocities vectors, ${ }^{21}$ as can be observed in Fig. 6(a). The use of a small $\epsilon$ fixes that behavior, as depicted in Fig. 6(b)

\section{Numerical Simulation of Flow in Thermochemical Nonequilibrium over a Mars Entry Vehicle}

This section studies the full configuration of the Mars entry vehicle experimental model simulated in the last section. The freestream and wall conditions for the flow are the same as in the last section. The mesh used in the computation is generated using GAMBIT. The mesh is made of quadrilaterals only, even though the code has the capability to use triangles in a $2 \mathrm{~d}$ simulation. Generating a good mesh using only quadrilaterals for this configuration is difficult. It is necessary to divide the domain into many blocks in order to ensure 


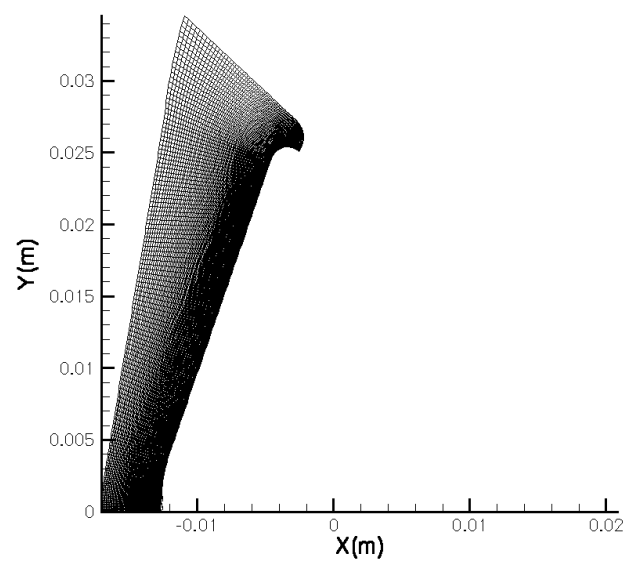

Figure 4. Mars entry vehicle model forebody mesh.

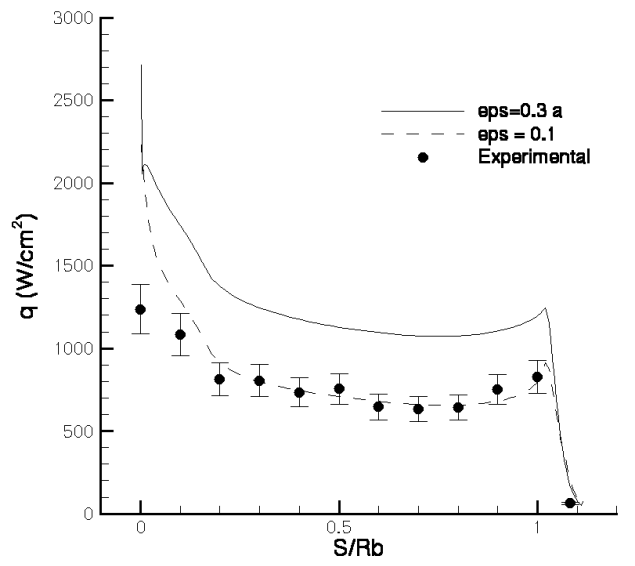

Figure 5. Heat transfer calculated using different pressure switches.

12 of 18 


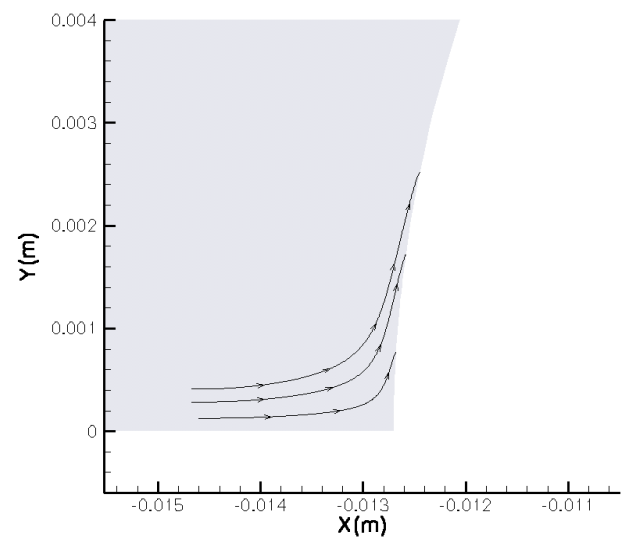

(a) $\epsilon=0.3 a$.

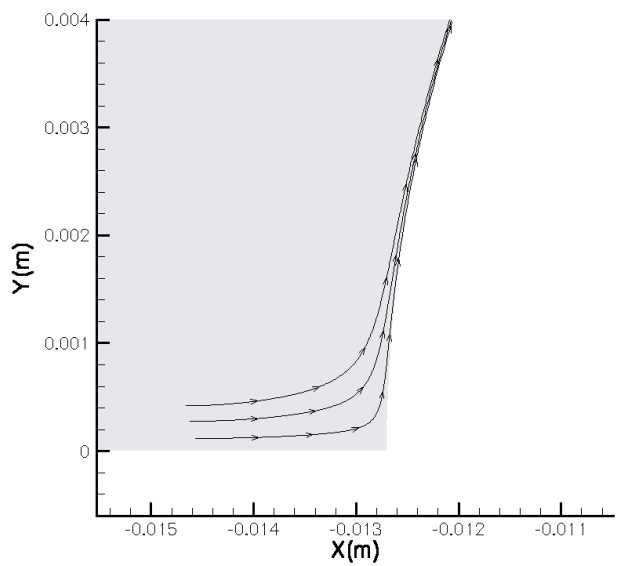

(b) $\epsilon=0.1$.

Figure 6. Streamtraces close to the body surface.

smooth mesh growth and to avoid abrupt changes in mesh orientation. The block division used in this work has 9 blocks and is shown in Fig. 7(a). The generated mesh is in Fig. 7(b). It has 200 cells in the direction normal to the body and 340 cells in the streamwise direction, totaling 68000 cells.

Figure 8(a) shows the flow pattern around the model. The main features in the flow are the detached bow shock in front of the model, the expansion wave formed on the shoulder of the model and a strong recirculation zone with 3 vortices that covers most of the leeside of the model and part of the sting. The detachment point is located just after the shoulder of the model. The flow pattern is in good agreement with the numerical results in Ref. 25 for the same configuration.

The level of nonequilibrium in the flow can be evaluated from Fig. 9, which plots the ratio of the translational-rotational to the vibrational temperature, $T_{t} / T_{v}$. From the figure, this ratio can reach values as high as 3.6 in the shock wave and as low as 0.3 inside the expansion fan and close to the body on the leeside. This behavior is further highlighted in Fig. 10(a), which shows the temperatures along the stagnation streamline. The maximum temperature is around $11000 \mathrm{~K}$ due to the high freestream Mach number and high freestream temperature. The vibrational temperature lags the translational-rotational temperature. It does not achieve very high temperatures because of the lag and air dissociation which starts to occur immediately after the shock wave.

A closer look at the air dissociation is provided in Figs. 11(a) and 11(b), which show the species mass density of $O$ and $N$, respectively. In both figures it is possible to observe the sudden rise of the species density after the shock due to the formation of this species in the dissociation process. These figures also show a decrease in $N$ and $O$ density close to the body, a sign that there is strong recombination inside the boundary layer. The recombination in the boundary layer releases energy and, therefore, increases the heat transfer to the wall. It should be noted that the wall is considered non-catalytic. The recombination happens inside the boundary layer and is due to the decrease in temperature provided by the low wall temperature.

The recombination in the boundary layer is also evident in Fig. 10(b). This figure presents the species mass fractions along the stagnation streamline. In this figure it is possible to see that $\mathrm{O}_{2}$ starts to dissociate slighty earlier than $N_{2}$ and that it is almost totally dissociated after the shock. On the other hand, $N_{2}$ does not fully dissociate due to the temperature after the shock not being high enough. The mass fractions of $N$ 


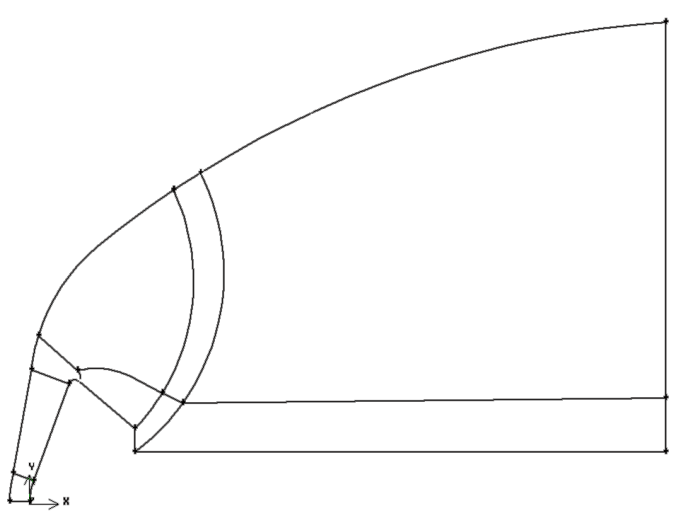

(a) Block structure

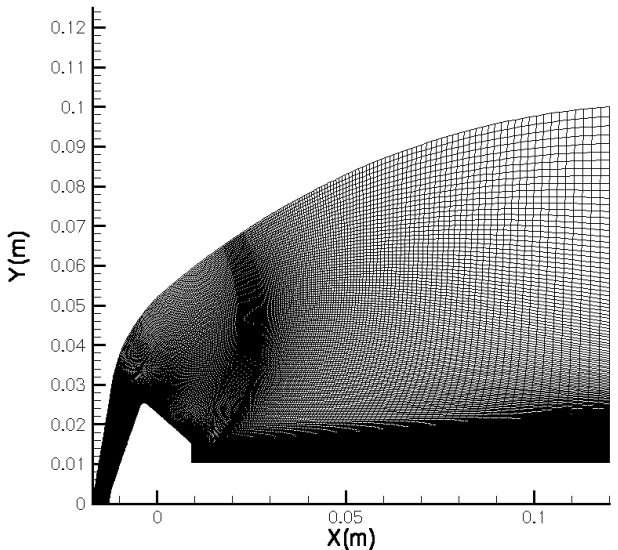

(b) Mesh

Figure 7. Block structure and mesh used in the computations.

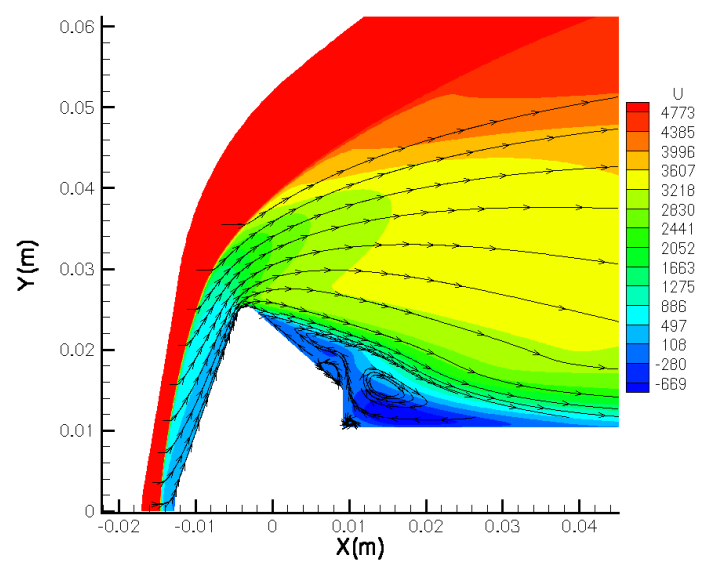

(a) x-velocity contours $(\mathrm{m} / \mathrm{s})$.

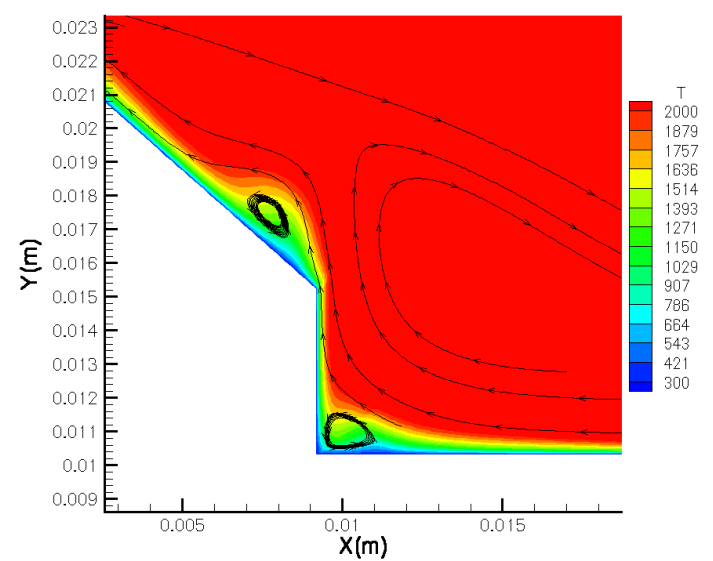

(b) Temperature contours (K) - Details at corners.

Figure 8. Flow pattern around model. 


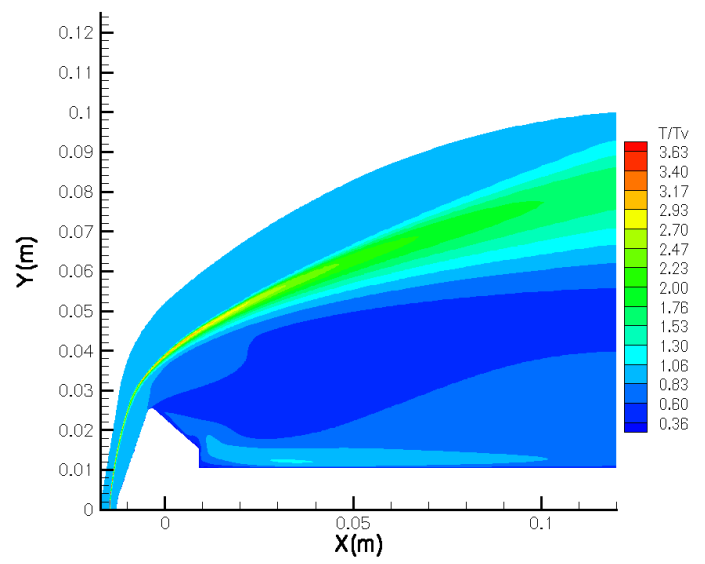

Figure 9. Ratio of translational-rotational to vibrational temperatures.

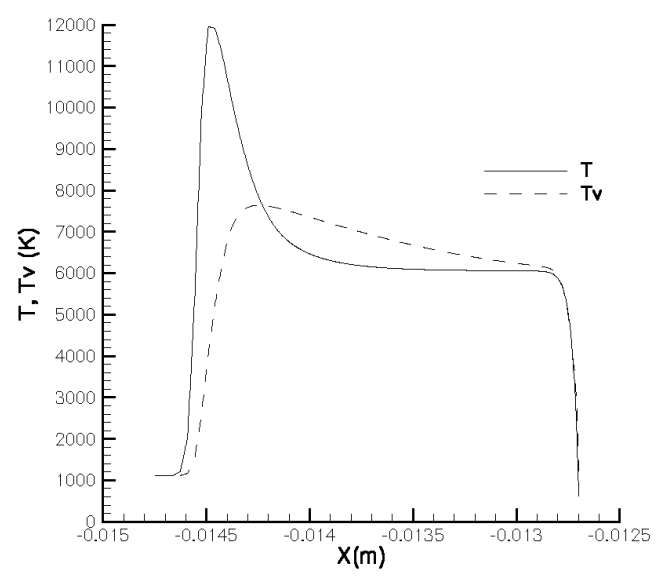

(a) Temperatures.

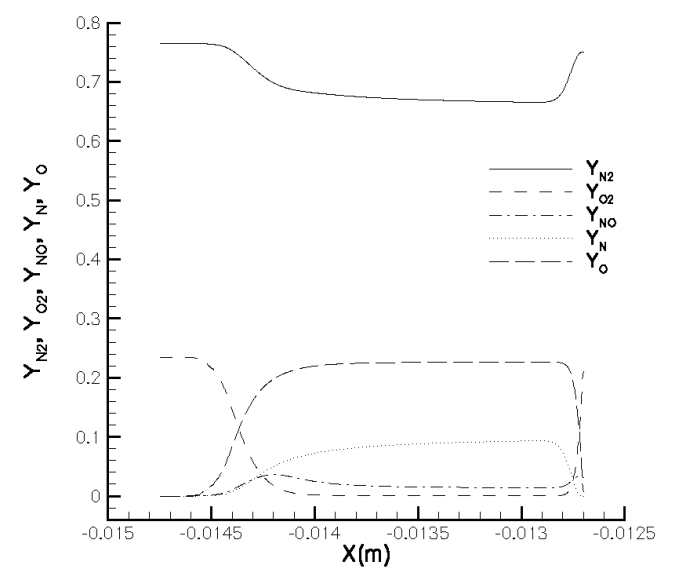

(b) Mass fractions.

Figure 10. Properties along stagnation streamline. 


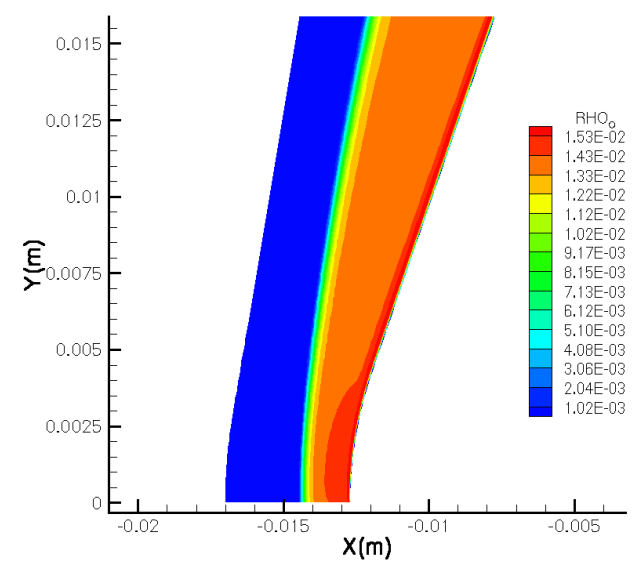

(a) Oxygen.

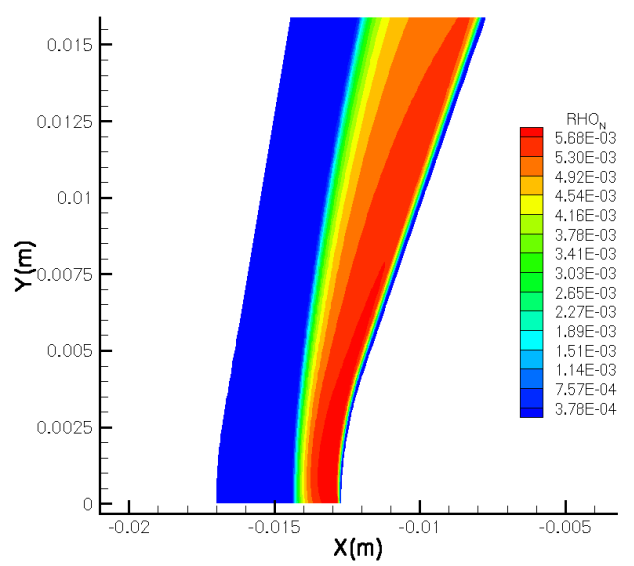

(b) Nitrogen.

Figure 11. Species density $\left(\mathrm{kg} / \mathrm{m}^{3}\right)$ over the model forebody.

and $O$ rapidly decrease inside the boundary layer due to the decrease in the temperature, forming $N_{2}, O_{2}$ and $N O$.

Results for heat transfer are compared to the experimental results of Refs. 26 and 24 . The comparison for the forebody is essentialy the same as that presented in Fig. 5. The results are in good agreement except for the stagnation region, as discussed previously. The comparison for the afterbody is shown in Fig. 12. The figure shows that the code is able to capture the overall shape of the heat distribution, but the agreement in detail is not very good. The location and value for the maximum heat transfer do not match the experiment. The numerical results present oscillations between $S / R b=1.7$ and $S / R b=2.0$, which corresponds to the back wall of the model. It is not clear at this moment if those oscillations are due to the numerical scheme or if they are due to the complex flow pattern in that region. For instance, the peak in heat transfer corresponds to where the large vortex impinges on the corner of the back wall squeezing the temperature profile in that region, as can be seen in Fig. 8(b). This causes an increase in the temperature gradient and, therefore, in the heat transfer. It is expected that a more accurate simulation using a second order scheme will improve the results for the afterbody. Also, the flow in the wake is very sensitive ${ }^{25}$ to grid resolution and a better mesh may be needed in this flow region.

\section{Concluding Remarks}

The paper showed the progress achieved in implementing a state of the art Navier-Stokes code capable of simulating flow in thermochemical nonequilibrium using unstructured meshes. The code models the fluid as a reacting gas in chemical and thermal nonequilibrium using standard finite-rate chemistry models and a two-temperature model for the gas. The code solves the set of differential equations using the finite volume technique. The fluxes across faces are calculated using a modified form of the Steger-Warming FVS which is switched back to the original form in the vicinity of shock waves. Time integration is performed using a point implicit method.

It was observed in this work that the switching between the modified and the original Steger-Warming FVS schemes has an influence on the amount of extra dissipation needed to stabilize the method. This work 


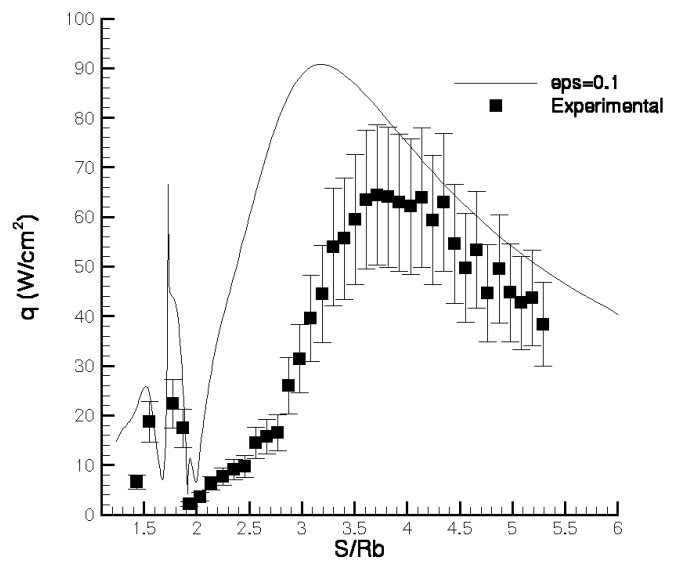

Figure 12. Heat transfer on the model afterbody.

proposed a new pressure switch which does not need any extra dissipation added because it effectively uses the original form of the Steger-Warming FVS in the vicinity of shocks. A way of improving the behavior of the code in axisymmetric calculations was also presented by modifying the approximation used to calculate the area of a cell.

The paper presented simulation results for an experimental model of a Mars entry vehicle for which measured heat transfer results are available. The comparisons between the numerical results obtained using the present code are good in the forebody region, except for the stagnation region where the numerical results show an unphysical peak. The reasons for the peak are not clear at this moment and further investigation is needed. The comparisons for the afterbody region are not as good. The numerical results predicted the wrong location and value for the maximum heat transfer. The results also present oscillations in the back side of the model, which are believed to be effects of the complex flow geometry in that region. However, given the early stages in the code development, numerical oscillations can not be discarded as causes of them. Further studies using 2nd order schemes and better meshes are needed in that region.

\section{Acknowledgments}

The authors are indebted to the Francois-Xavier Bagnoud Foundation which provides a fellowship to the first author. Additional funding is provided by the Space Vehicle Technology Institute, under NASA grant NCC3-989 with joint sponsorship from the Department of Defense. The authors are thankful to Prof. Graham Candler, Dr. Ioannis Nompelis and Travis Drayna for valuable discussions and material on the subject. The authors also would like to thank Prof. Van Leer for numerous discussions on numerical schemes and Dr. Brian Hollis for providing the experimental results used in the comparisons.

\section{References}

\footnotetext{
${ }^{1}$ Wang, W.-L. and Boyd, I. D., "Hybrid DSMC-CFD Simulations of Hypersonic Flow over Sharp and Blunted Bodies," AIAA Paper 2003-3644, 2003.

${ }^{2}$ Wang, W.-L. and Boyd, I. D., "A New Energy Flux Model in the DSMC-IP Method for Nonequilibrium Flows," AIAA Paper 2003-3774, 2003.

${ }^{3}$ Wang, W.-L. and Boyd, I. D., "Predicting Continuum Breakdown in Hypersonic Viscous Flows," Physics of Fluids, Vol. 15, Jan. 2003, pp. 91-100.
} 
${ }^{4}$ Schwartzentruber, T. E. and Boyd, I. D., "Detailed Analysis of a Hybrid CFD-DSMC method for Hypersonic NonEquilibrium Flows," AIAA Paper 2005-4829, 2005.

${ }^{5}$ Lee, J. H., "Basic Governing Equations for the Flight Regimes of Aeroassisted Orbital Transfer Vehicles," Thermal Design of Aeroassisted Orbital Transfer Vehicles, Vol. 96, AIAA, New York, 1985, pp. 3-53.

${ }^{6}$ Gnoffo, P. A., Gupta, R. N., and Shinn, J. L., "Conservation Equations and Physical Models for Hypersonic Air Flows in Thermal and Chemical Nonequilibrium," NASA-TP-2867, NASA Langley, Hampton, Virginia, 1989.

${ }^{7}$ Park, C., Nonequilibrium Hypersonic Aerothermodynamics, John Wiley \& Sons, 1990.

${ }^{8}$ Hirsch, C., Numerical Computation of Internal and External Flows, John Wiley \& Sons, 1991.

${ }^{9}$ MacCormack, R. W. and Candler, G. V., "The Solution of the Navier-Stokes Equations Using Gauss-Seidel Line Relaxation," Computers and Fluids, Vol. 17, 1989, pp. 135-150.

${ }^{10}$ Steger, J. L. and Warming, R. F., "Flux Vector Splitting for the Inviscid Gasdynamic Equations with Applications to Finite Difference Methods," Journal of Computational Physics., Vol. 40, 1981, pp. 263-293.

${ }^{11}$ Jawahar, P. and Kamath, H., "A High-Resolution Procedure for Euler and Navier-Stokes Computations on Unstructured Grids," Journal of Computational Physics, Vol. 164, 2000, pp. 165-203.

${ }^{12}$ Wright, M. J., A Family of Data-Parallel Relaxation Methods for the Navier-Stokes equations, Ph.D. thesis, University of Minnesota, Minnesota, June 1997.

${ }^{13}$ Gnoffo, P. A., "Computational Aerothermodynamics in Aeroassist Applications," Journal of Spacecrafts and Rockets, Vol. 40, No. 3, 2003, pp. 305-312.

${ }^{14}$ Venkatakrishnan, V., "Implicit Schemes and Parallel Computing in Unstructured Grid CFD," VKI-LS-1995-02, VKI Lecture Series, 1995.

${ }^{15}$ Nompelis, I., Computational Study of Hypersonic Double-Cone Experiments for Code Validation, Ph.D. thesis, University of Minnesota, Minnesota, May 2004.

${ }^{16}$ Wilke, C. R., "A Viscosity Equation for Gas Mixtures," J. of Chem. Phys., Vol. 18, 1950, pp. 517-519.

${ }^{17}$ Blottner, F. G., Johnson, M., and Ellis, M., "Chemically Reacting Viscous Flow Program for Multi-Component Gas Mixtures," Sc-rr-70-754, Sandia Laboratories, Albuquerque, New Mexico, 1971.

${ }^{18}$ Vincenti, W. G. and Kruger, C. H., Introduction to Physical Gas Dynamics, Krieger Publishing Company, 1982.

${ }^{19}$ Millikan, R. C. and White, D. R., "Systematics of Vibrational Relaxation," J. of Chem. Phys., Vol. 39, 1963, pp. 32093213.

${ }^{20}$ Buning, P. G. and Steger, J. L., "Solution of the Two-Dimensional Euler Equations with Generalized Coordinate Transformations Using Flux Vector Splitting," AIAA Paper 82-0971, 1982.

${ }^{21}$ Druguet, M., Candler, G. V., and Nompelis, I., "Effect of Numerics on Navier-Stokes Computations of Hypersonic Double-Cone Flows," AIAA Journal, Vol. 43, No. 3, 2005, pp. 616-623.

${ }^{22}$ Nompelis, I., Drayna, T. W., and Candler, G. V., "Development of a Hybrid Unstructured Implicit Solver for the Simulation of Reacting Flows Over Complex Geometries," AIAA Paper 2004-2227, 2004.

${ }^{23}$ Leer, B. V. and Mulder, W. A., "Relaxation Methods for Hyperbolic Conservation Laws," Numerical Methods for the Euler Equations of Fluid Dynamics, Vol. 21 of Proceedings in Applied Mathematics, SIAM, Philadelphia, 1985, pp. 312-333.

${ }^{24}$ Hollis, B. R. and Perkins, J. N., "High-Enthalpy Aerothermodynamics of a Mars Entry Vehicle Part 1: Experimental Results," Journal of Spacecraft and Rockets, Vol. 34, No. 4, 1997, pp. 449-456.

${ }^{25}$ Hollis, B. R. and Perkins, J. N., "High-Enthalpy Aerothermodynamics of a Mars Entry Vehicle Part 2: Computational Results," Journal of Spacecraft and Rockets, Vol. 34, No. 4, 1997, pp. 457-463.

${ }^{26}$ Hollis, B. R., "Experimental and Computational Aerothermodynamics of a Mars Entry Vehicle," NASA-CR-201633, Dec. 1996.

18 of 18 